\title{
Disorder in van der Waals heterostructures of 2D materi- als
}

Daniel Rhodes ${ }^{1, *}$, Sang Hoon Chae ${ }^{1, *}$, Rebeca Ribeiro-Palau ${ }^{2}, \&$ James Hone $^{1}$

${ }^{1}$ Department of Mechanical Engineering, Columbia University, New York, NY, USA

${ }^{2}$ Centre de Nanosciences et de Nanotechnologies (C2N), CNRS, Université Paris Sud, Université Paris-Saclay, 91120 Palaiseau, France.

${ }^{*}$ These authors contributed equally to this work.

Realizing the full potential of any materials system requires understanding and controlling sources of disorder that can obscure intrinsic properties and hinder device performance. Here we examine progress in understanding and reducing both intrinsic and extrinsic disorder in two-dimensional (2D) graphene and transition metal dichalcogenides (TMDs) incorporated into layered van der Waals heterostructures. In the case of mechanically exfoliated graphene, the dominant disorder is extrinsic and can be reduced by encapsulation within crystalline hexagonal boron nitride ( $h \mathrm{BN})$. Continuing improvement in encapsulation and device fabrication techniques has led to dramatic performance improvement and enabled the study of a wide range of novel phenomena. In the case of TMDs, both extrinsic and intrinsic disorder are relevant. The electronic and optical performance of semiconducting TMDs improves with the reduction of external disorder, but commonly used materials show high defect density which must be reduced to enable similar progress as demonstrated in graphene. These atomic defects can also be harnessed to provide useful electronic, optical, 


\section{chemical, and magnetic properties.}

Atomically thin 2D materials have generated wide interest for electronic and optoelectronic applications, and as hosts for novel physical phenomena such as topologically protected states, valley effects, moiré physics, and exciton superfluidity. Toward these goals, minimizing disorder is crucial in order to reveal intrinsic properties and improve device performance and repeatability. A particular challenge is that 2D systems are by definition entirely surface, and therefore highly susceptible to external sources of disorder. Thus the challenge of controlling disorder in 2D materials includes both intrinsic disorder arising from crystalline imperfections, and extrinsic disorder arising from the environment. Below we review progress toward this goal in two specific systems: mechanically exfoliated graphene, in which intrinsic disorder is minimal and dramatic improvement is seen with encapsulation by $h \mathrm{BN}$ in a van der Waals heterostructure; and semiconducting TMDs for the intrinsic crystal quality must also be addressed in order to achieve similar progress.

Figure 1 summarizes the main sources of intrinsic and extrinsic disorder in $2 \mathrm{D}$ materials. Sources of extrinsic disorder, depicted in Fig. 1c, include: strain, adsorbates, surface roughness, charged impurities in the substrate, and oxidation. These sources of disorder can affect multiple properties by introducing electrostatic potential variation, scattering of charge carriers ${ }^{4}$, locally modifying the electronic band structure ${ }^{5}$, and acting as recombination centers for excitons ${ }^{6}$. Sources of intrinsic disorder (depicted in Fig. 1a for a hexagonal lattice but applicable to all 2D crystals), include atomic defects such as vacancies, anti-sites, substitutions, edges, and grain boundaries. Such grain boundaries are common in large area, polycrystalline monolayer films (see

Fig. 1b, lower panel), and can dominate the materials' properties. In graphene, transport across a 
a

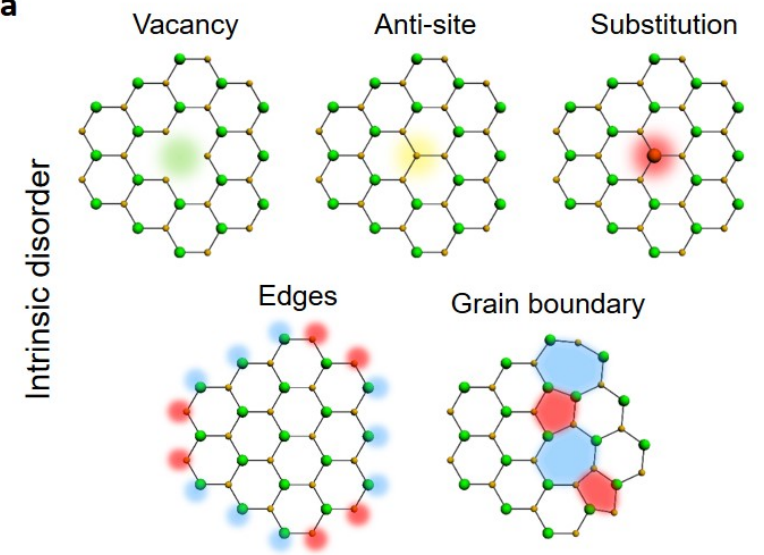

C

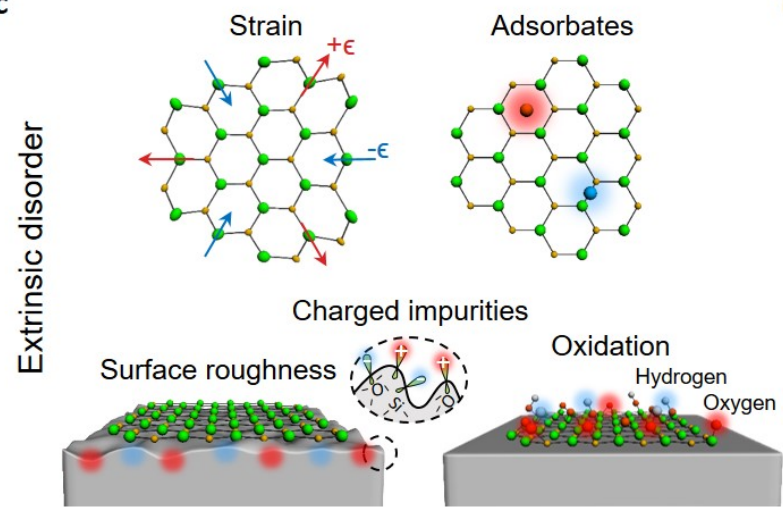

b
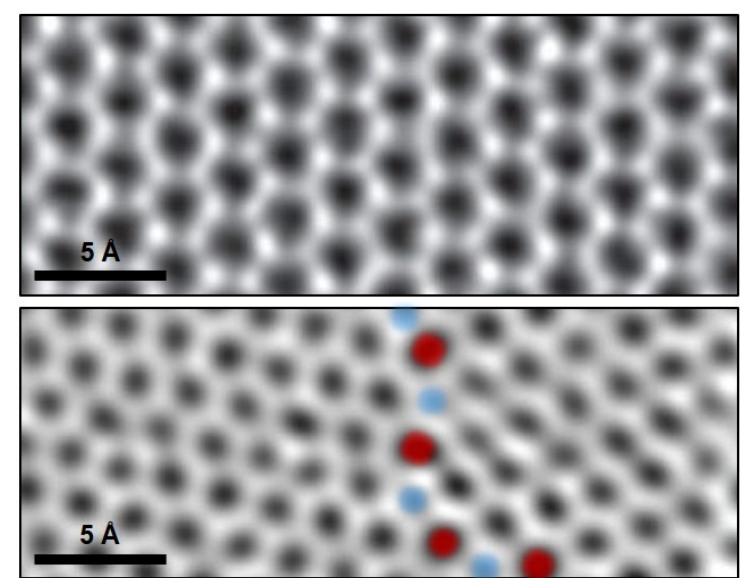

d

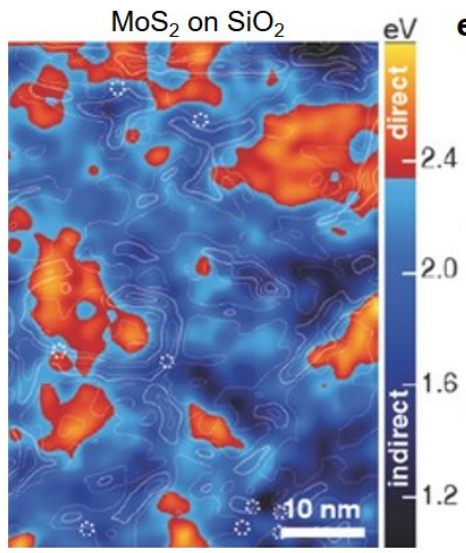

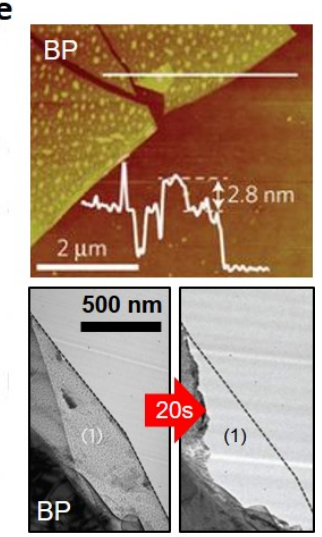

Figure 1 | Types of disorder in 2D materials. a, Intrinsic disorder: vacancy, anti-site, substitution, edges termination, and grain boundary. $\mathbf{b}$, Aberration-corrected annular dark-field scanning transmission electron microscopy image of graphene lattice ${ }^{1}$ showing absence of defects (top) and grain boundary of CVD-grown graphene (bottom). c, Extrinsic disorder: strain, adsorbates, surface roughness, charged impurities in the substrate, and oxidation. d, STS map of the bandgaps in $\mathrm{MoS}_{2}$ on an $\mathrm{SiO}_{2}$ substrate ${ }^{2}$. e, AFM (top) and TEM (bottom) images of black phosphorus (BP) under oxidation. After $20 \mathrm{~s}$ exposure, the suspended region (1) has collapsed ${ }^{3}$. Figure reproduced with permission: adapted from ref. ${ }^{1}$, Nature Publishing Group (b); ref. $^{2}$, Wiley (d); ref. ${ }^{3}$, Nature Publishing Group (e). 
single grain boundary can decrease conductivity by a factor of three ${ }^{7}$. In TMDs, grain boundaries can produce local strain that can either reduce or increase the band gap depending on the tilt angle between each grain ${ }^{8}$. The role of defects and grain boundaries in 2D materials has been reviewed in detail ${ }^{9}$. In order to understand how electronic properties depend on other types of disorder, it is important to investigate single crystalline material. Below, we will continue to discuss intrinsic disorder in the context of single crystal monolayers produced through mechanical exfoliation.

Mechanically exfoliated graphene provides a case study for a material in which virtually most of the disorder is extrinsic. Figure $1 \mathrm{~b}$ shows an annular dark-field scanning transmission electron microscopy (ADF-STEM) image of the graphene lattice showing the absence of any vacancies or Stone-Wales defects ${ }^{1}$. In fact, extensive investigation by transmission electron microscopy (TEM) and scanning tunnelling microscopy (STM) have shown that graphene flakes obtained by mechanical exfoliation from bulk graphite crystals are remarkably free of defects ${ }^{1}$. In spite of the high quality of mechanically exfoliated graphene, early devices fabricated on $\mathrm{SiO}_{2}$ substrates showed evidence of substantial disorder in electrical transport studies ${ }^{10}$. This disorder can be directly visualized by mapping the local potential variation using a scanning single electron transistor ${ }^{11}$ or by scanning tunnelling spectroscopy (STS) ${ }^{12,13}$. For charge-neutral graphene on $\mathrm{SiO}_{2}$, chemical potential variations of $\sim 100 \mathrm{meV}$ (corresponding to carrier density variations of $\delta n \sim 10^{12} \mathrm{~cm}^{-2}$ ) on the length scale of tens of nanometers can be readily observed. These electron-hole 'puddles' have been shown to arise from trapped charge impurities in the $\mathrm{SiO}_{2}$ substrate ${ }^{14}$ and surface adsorbates. Theoretical and experimental analysis indicates that scattering from this electrical potential disorder provides the dominant scattering mechanism for charge carriers in graphene at low tempera- 
ture, providing the connection between charge disorder and electronic mobility ${ }^{15,16}$. Roughness of $\mathrm{SiO}_{2}$ can introduce strain variation in the 2D system at similar length scales which cause charge fluctuations $^{12}$. In TMDs, bending strain induced by surface roughness, which strongly modifies the local bandstructure, is likely the dominant source of disorder on $\mathrm{SiO}_{2}$ substrates. Figure 1d shows a color map for the bandgap of monolayer $\mathrm{MoS}_{2}$ on $\mathrm{SiO}_{2}$, characterized by scanning tunneling spectroscopy $(\mathrm{STS})^{2}$. Significant modulations in the bandgap, 1.23-2.65 eV, are observed with $\sim 80 \%$ of the $\mathrm{MoS}_{2}$ under sufficient enough strain to cause a direct-to-indirect bandgap transition.

Oxidation is another source of extrinsic disorder in many 2D materials that, unlike graphene, are air-sensitive ${ }^{17}$. A prime example is semiconducting black phosphorus (BP): small bumps of adsorbed water are seen by atomic force microscopy (AFM) immediately after exfoliation in ambient conditions (top panel of Fig. $1 \mathrm{e}^{3}$ ), and TEM imaging shows that few-layer BP completely collapses after $20 \mathrm{~s}$ of exposure to air (bottom panel of Fig. 1e). Recent work has shown that a photoassisted oxidation reaction by oxygen dissolved in adsorbed water is the dominant mechanism for degradation ${ }^{3}$.

A straightforward and effective way to reduce disorder arising from the substrate is to suspend the $2 \mathrm{D}$ layer so that it is not in contact with the $\mathrm{SiO}_{2}$. Indeed, a study of suspended graphene ${ }^{18}$ has shown dramatically increased carrier mobility and greatly reduced charge inhomogeneity, reaching a residual carrier density $\delta n \sim 2 \times 10^{9} \mathrm{~cm}^{-2}$. This lower disorder permits the observation of fractional quantum Hall $(\mathrm{QH})$ states $^{19,20}$, interaction-driven ground states ${ }^{21}$, and hydrodynamic phenomena $^{22}$. However, suspended devices are limited in important ways: they are limited to low carrier density and weak gate capacitance, complex geometries are extremely challenging, and 
multilayer structures are impossible. Therefore, a major goal in the field is to create substratesupported devices with charge disorder (i.e. $\delta n)$ approaching the cleanest suspended graphene. Toward this end, the most successful approach to date utilizes hexagonal boron nitride $(h \mathrm{BN})$, a layered wide-bandgap $(\sim 6 \mathrm{eV})$ insulator ${ }^{23}$ with a crystalline structure similar to graphene, as a replacement for conventional dielectrics such as $\mathrm{SiO}_{2}$. Below we examine the effects of $h \mathrm{BN}$ encapsulation on disorder and materials performance, first for graphene and then for TMDs.

\section{Reducing extrinsic disorder in graphene- $h \mathrm{BN}$ heterostructures}

$60 \mathrm{~nm} \times 60 \mathrm{~nm}$ STM topography (Fig. 2a) and charge density (Fig. 2b) maps of monolayer graphene on $h \mathrm{BN}$ and $\mathrm{SiO}_{2}$ substrates show the clear advantage of $h \mathrm{BN}^{13}$. Roughness in the graphene/ $h \mathrm{BN}$ charge density for the surface $\left(2.3 \times 10^{10} \mathrm{~cm}^{-2} \mathrm{rms}\right)$ is much lower than what is observed for graphene/ $\mathrm{SiO}_{2}\left(8.2 \times 10^{10} \mathrm{~cm}^{-2} \mathrm{rms}\right)$. Both the roughness and charge density inhomogeneities are dramatically reduced on $h \mathrm{BN}$ substrates, making $h \mathrm{BN}$ an ideal candidate for supporting 2D materials $\mathrm{s}^{12,13,24}$.

Figure $2 \mathrm{c}$ depicts the evolution of graphene devices as continued improvements to fabrication techniques have reduced extrinsic disorder. These are divided into four approximate 'generations', beginning with device fabricated on $\mathrm{SiO}_{2}{ }^{10}$ (Generation 1). The first graphene- $h \mathrm{BN}$ devices $^{24}$ (Generation 2) were assembled by using polymers to pick up graphene flakes and place them onto $h \mathrm{BN}$. Although these devices showed lower disorder than those on $\mathrm{SiO}_{2}{ }^{12,13}$, they remained affected by polymer residue from the transfer and lithographic processing. In addition, this residue can impede assembly of multilayer structures by aggregating into 'bubbles' between layers. Improved performance was seen upon the removal of residue (by annealing in oxygen or 
a
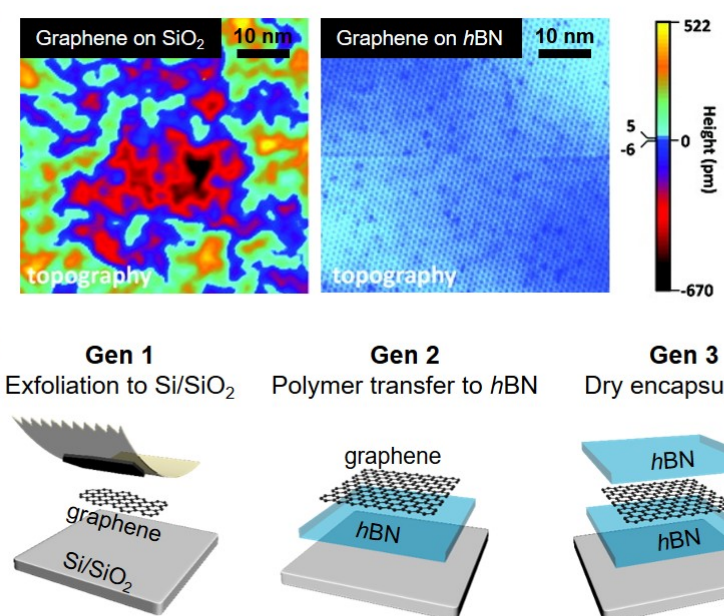

e

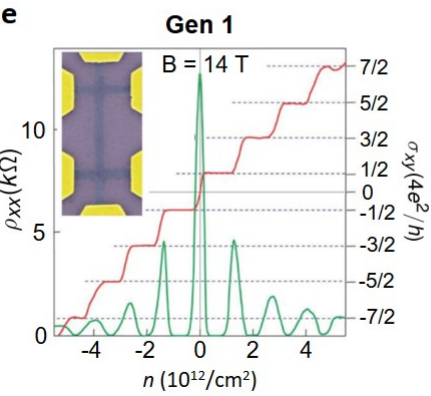

b
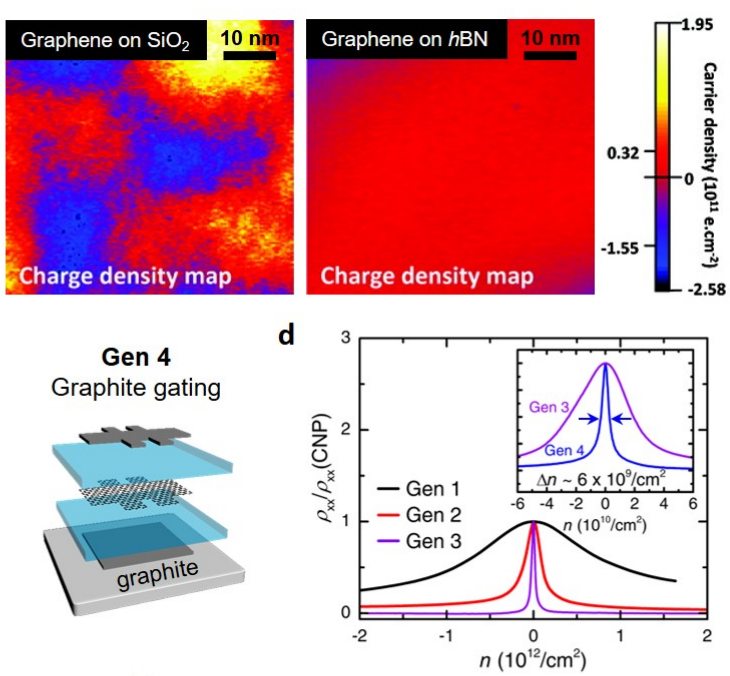

g f

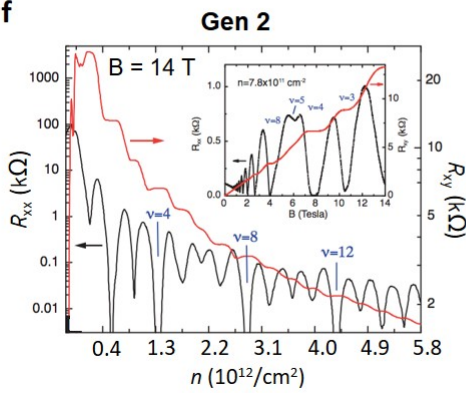

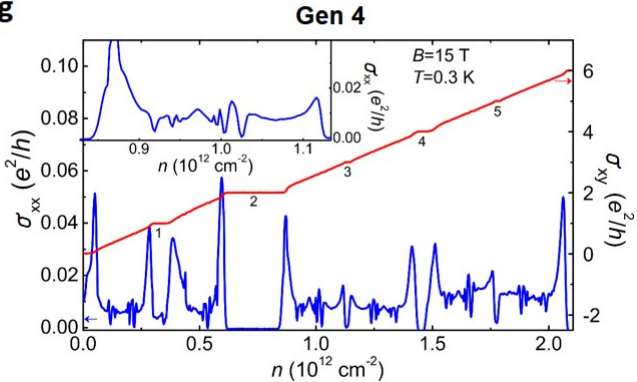

Figure 2 | Evolution of graphene device design and resulting performance improvements. Compar-

ing STM topography (a) and charge density maps (b) for graphene/hBN vs graphene $/ \mathrm{SiO}_{2}{ }^{13}$. c, Device fabrication techniques ( $\mathrm{L}-\mathrm{R})$ : Generation 1, mechanical exfoliation onto $\mathrm{Si} / \mathrm{SiO}_{2}$ substrates; Generation 2, graphene transfer onto $h \mathrm{BN}$ using a polymer film; Generation 3, dry encapsulation in $h \mathrm{BN}$ by a van der Waals layer assembly technique; Generation $4, h \mathrm{BN}$ encapsulation with graphite gates. d, Normalized resistivity vs gate-induced charge density for devices of Generations $1-4$, showing the dramatic decrease in Dirac peak width due to reduction of disorder-induced broadening. Quantum Hall effect measurements, for similar magnetic fields and for: e, Generation 1 device, showing four-fold degenerate integer states ${ }^{10} ; \mathbf{f}$, Generation 2 device showing all integer $\operatorname{states}^{24}, \mathbf{g}$, Generation 4 device showing well resolved fractional quantum Hall states. Adapted from ref. ${ }^{13}$, ACS Publications (a,b); ref. ${ }^{10}$, Nature Publishing Group (e); ref. ${ }^{24}$, Nature Publishing Group (f). 
by 'sweeping' with an AFM tip ${ }^{25}$ ) and in micron-scale $h \mathrm{BN}$-encapsulated graphene ${ }^{26}$. Large-area $h \mathrm{BN}$-encapsulated devices (Generation 3) are assembled by using the top layer in the heterostructure to 'pick up' subsequent layers, using only van der Waals forces, so that the interior surfaces are never in contact with the polymers ${ }^{27}$ (Generation 3). The graphene- $h \mathrm{BN}$ interfaces produced by this van der Waals assembly technique show no evidence of contamination in cross-sectional imaging by scanning transmission electron microscopy (STEM), and bubble-free areas tens of $\mu \mathrm{m}$ in size can be achieved; electrical contact to the encapsulated graphene is achieved by metalization of the graphene edge exposed after etching the assembled heterostructure ${ }^{27}$. These structures reduce, but do not eliminate, the effects of charge disorder in the underlying $\mathrm{SiO}_{2}$ substrate, and intervening metal gates used to eliminate these effects can introduce variations in the electrostatic potential as well ${ }^{28}$. The lowest disorder is instead achieved by utilizing graphite gates ${ }^{29}$ (Generation 4). Local potential variation around metal contacts is also minimized by using graphite for electrical contacts, in essence creating devices entirely from van der Waals materials.

A simple yet accurate technique to estimate charge inhomogeneity in graphene devices is to measure the width of the peak in resistance as a function of gate-induced carrier density across the charge neutrality point $(\mathrm{CNP})^{16,30}$. A more rigorous method is to directly measure carrier density by Hall effect, but the two approaches give very similar results ${ }^{31}$. The CNP resistance peaks for four generations of graphene devices are shown in Fig. 2d, showing a dramatic narrowing from $\delta n \sim 10^{12} \mathrm{~cm}^{-2}$ (Gen 1) to $6 \times 10^{9} \mathrm{~cm}^{-2}(\mathrm{Gen} 4)-$ within a factor of three of the lowest reported for suspended graphene ${ }^{20}$. Similar estimates of charge disorder are obtained by STM mapping ${ }^{12,13}$. At this point the origin of the remaining disorder is unclear; small amounts of environmental impu- 
rities such as water or hydrocarbons may still be present at the graphene- $h \mathrm{BN}$ interface, and charge disorder may arise from oxygen or carbon impurities in the $h \mathrm{BN}$, which occur at concentrations similar to the charge fluctuations seen in $\mathrm{STM}^{23}$. Beyond measurement of disorder by CNP peak width, electron transport measurements can also be used to extract the carrier mobility, which increases as $\delta n$ decreases. Gen 3 and 4 devices show ballistic conduction, with an electronic mean free path limited by device size (typically $<20 \mu \mathrm{m}$ ) rather than impurity scattering, which provides a lower limit of $\sim 10^{6} \mathrm{~cm}^{2} \mathrm{~V}^{-1} \mathrm{~s}^{-1}$ (at $n=10^{12} \mathrm{~cm}^{-2}$ ) on the intrinsic carrier mobility ${ }^{27,32}$. A more accurate determination of the intrinsic mobility would require assembly of devices on the scale of $100 \mu \mathrm{m}$, which is currently difficult given the size of mechanically exfoliated $h \mathrm{BN}$ flakes.

The reduction of disorder in successive generations of graphene devices is better reflected in more sensitive measurements such as the quantum Hall effect (QHE). Gen 1 devices (Fig. 2e) show four-fold degenerate integer states, with the sequence famously reflecting the Berry phase ${ }^{10}$. Gen 2 devices (Fig. 2f), under the same experimental conditions, show breaking of the four-fold symmetry $^{24}$, reflecting the improved quality. In fully-encapsulated structures (Gen 3), at high magnetic fields ${ }^{33}$ further details of the fractional quantum Hall effect (FQHE) can be observed. In Gen 4 devices (Fig. 2g) well developed FQHE can be observed from magnetic fields as low as $6 \mathrm{~T}^{34}$. In particular, Gen 4 devices that probe bulk insulating states capacitively ${ }^{35}$ or through transport measurements in a Corbino geometry ${ }^{36}$ show well developed integer QHE states emerging at magnetic fields as small as $B \sim 50 \mathrm{mT}$, symmetry-broken integer states at around $B=1 \mathrm{~T}$, and easily resolvable FQHE states by $B \sim 5 \mathrm{~T}$. When comparing to Gen 4 devices in the Hall bar geometry these results show that edges remain as a source of intrinsic disorder still to be overcome. 
Quantum transport measurements allow a more direct benchmark of 4th-generation graphene devices to GaAs/AlGaAs quantum wells with mobilities exceeding $3 \times 10^{7} \mathrm{~cm}^{2} \mathrm{~V}^{-1} \mathrm{~s}^{-1}$, which represent the 'gold standard' for low-disorder 2D electron gas (2DEG) devices ${ }^{37}$. In the low-field regime, Shubnikov-de Haas $(\mathrm{SdH})$ oscillations indicate a quantum scattering time $\tau_{q}$ of $0.2-0.3$ ps in graphene, whereas GaAs 2DEGs show values above $20 \mathrm{ps}^{38}$. Likewise, the Landau level broadening measured by measuring the activation gap state as a function of magnetic field yields $\Gamma \sim 10 \mathrm{~K}$, for edge $\mathrm{e}^{34}$ and edgeless ${ }^{36}$ graphene devices and $0.1-1 \mathrm{~K}$ for GaAs ${ }^{39}$. Thus the cleanest graphene devices still show roughly two orders of magnitude higher bulk disorder than GaAs. However, graphene stringer interactions strength and the ability to tune its carrier density enables the investigation and control of $\mathrm{QH}$ states. As a result, nearly every $\mathrm{QH}$ state originally observed in GaAs has now been seen in graphene (including recently-reported ordered electron solids ${ }^{34}$ ), with the notable exception of the 'stripe' phases ${ }^{40}$. Beyond QH physics, new regimes such as hydrodynamic transport are accessible in low-disorder graphene due to strong carrier-carrier interactions ${ }^{22}$. Moreover, graphene- $h \mathrm{BN}$ heterostructures offer greater flexibility in architecture versus conventional heterostructures. For instance, graphene layers can be placed extremely close to external patterned gates allowing strong bandstructure modification ${ }^{41}$, or to each other to study interaction effects in the strong coupling limit ${ }^{42}$. Likewise, the weak interlayer van der Waals interaction allows for the emergence of moiré superlattices that give rise to 'Hofstader Butterfly' physics in graphene- $h \mathrm{BN}$ devices ${ }^{43,44}$, and a strongly modified bandstructure in twisted bilayer graphene that can host both insulating and superconducting phases ${ }^{45}$. Therefore graphene- $h \mathrm{BN}$ heterostructures can be seen as a complementary materials system with quality still below that of GaAs, but with 
the benefit of providing access to a wide range of new physical phenomena.

\section{Extrinsic disorder in semiconducting transition metal dichalcogenides}

TMDs are compounds of the family $\mathrm{MX}_{2}$, where $\mathrm{M}$ is a transition metal (Nb, Mo, $\mathrm{W}$, etc.) and $\mathrm{X}$ is a chalcogen $(\mathrm{S}, \mathrm{Se}, \mathrm{Te})$. For this discussion, we will focus on monolayer semiconducting Mo- and W-based TMDs with a trigonal prismatic, $2 \mathrm{H}, \mathrm{P}_{3} / \mathrm{mmc}$ structure, akin to the honeycomb structure of graphene ${ }^{52}$. As with graphene, $\mathrm{SiO}_{2}$ substrates introduce substantial extrinsic disorder to monolayer TMDs. Two main approaches have been utilized to reduce the effects of this disorder: i) Deposition of high- $\kappa$ dielectric (i.e. $\mathrm{HfO}_{2}$ or $\left.\mathrm{Al}_{2} \mathrm{O}_{3}\right)^{53}$, which can both screen charged impurities and reduce charged impurity density by surface passivation; and ii) encapsulation within $h \mathrm{BN}^{48}$, which moves the conducting channel away from the $\mathrm{SiO}_{2}$ surface. Charge disorder from the $\mathrm{SiO}_{2}$ surface can also be screened by placing a conducting backgate under the $h \mathrm{BN}^{49}$. Reducing this extrinsic disorder improves electronic transport properties, as seen in measurements of carrier mobility and quantum transport measurements, as well as optical properties, as seen in photoluminescence spectra.

The electronic mobility of monolayer TMDs reflects the separate contributions of temperaturedependent scattering from phonons (both within the material and in the substrate) and static scattering from charge disorder - both are modulated by the dielectric environment and carrier density. Figure 3a shows theoretical calculations of the mobility for monolayer $\mathrm{MoS}_{2}$ at fixed electron density $n=1 \times 10^{13} \mathrm{~cm}^{-2}$ on $\mathrm{SiO}_{2}$ with $\mathrm{HfO}_{2}$ encapsulation ${ }^{4}$. At low-temperature, the mobility is entirely dominated by charged impurity scattering, varying inversely with impurity density $N_{i}$. At higher temperatures, scattering from optical phonons dominates the electronic transport behavior 

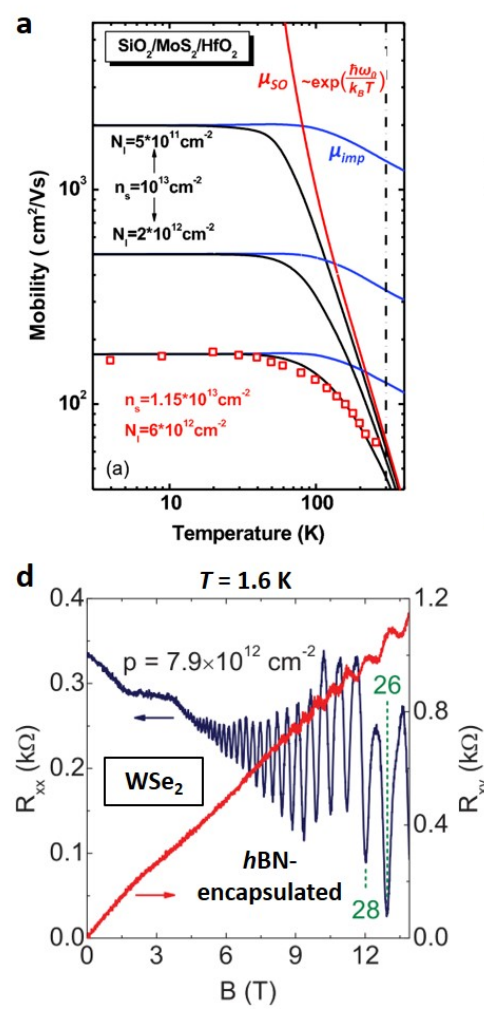

b

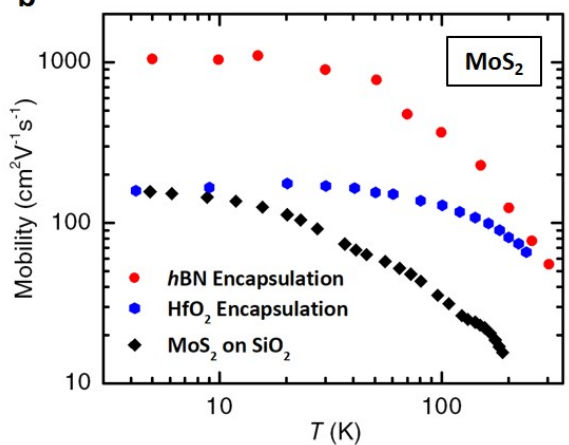

e

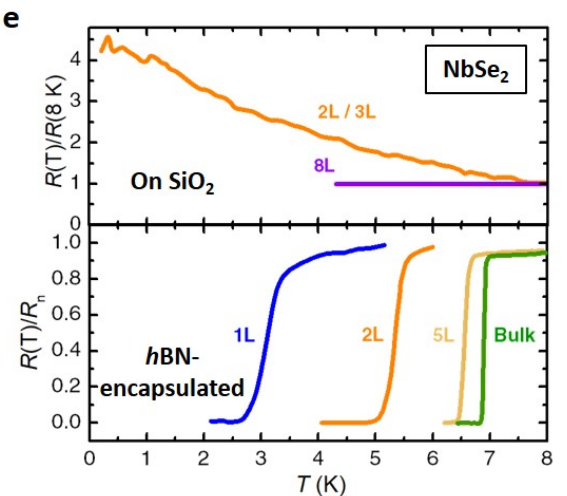

c

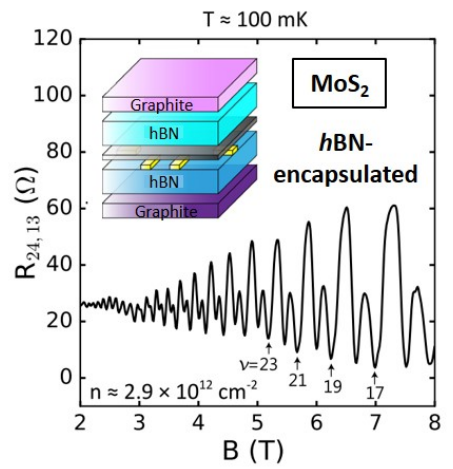

f

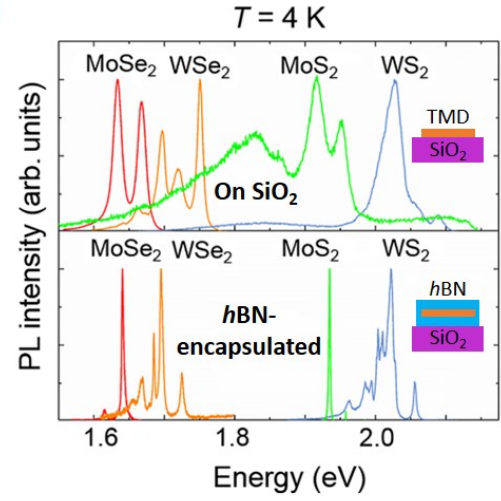

Figure 3 | Reducing extrinsic disorder in TMDs through $h \mathrm{BN}$ encapsulation. a, Theoretical calcula-

tion of temperature-dependent mobilities (black: electron mobility, blue: impurity mobility, red: phononscattering mobility) in $\mathrm{SiO}_{2} / \mathrm{MoS}_{2} / \mathrm{HfO}_{2}$ structure ${ }^{4}$. b, Experimental observation of mobility versus temperature on a log scale for monolayer $\mathrm{MoS}_{2}$ on $\mathrm{SiO}_{2}{ }^{46}, \mathrm{HfO}_{2}{ }^{47}$, and encapsulated in $h \mathrm{BN}^{48}$. c, $\mathrm{SdH}$ as a function of magnetic field in Gen 3 constructed monolayer $\mathrm{MoS}_{2}$ devices showing a clear valley Zeeman effect, i.e. weak/strong oscillations for even/odd states ${ }^{49}$. d, $\mathrm{SdH}$ as a function of magnetic field in monolayer $\mathrm{WSe}_{2}$ at $\mathrm{T} \sim 1.6 \mathrm{~K}$ and a hole density of $7.9 \times 10^{12} \mathrm{~cm}^{-2}$. Well defined SdH oscillations, and QH states in high magnetic fields with high-mobility holes are observed in monolayer $\mathrm{WSe}_{2}{ }^{50}$. e, Superconductivity behaviour of few-layered/bulk $\mathrm{NbSe}_{2}$ (directly exfoliated on $\mathrm{SiO}_{2}$ (top) and encapsulated by $h \mathrm{BN}$ (bottom)). Superconductivity is only observed in encapsulated samples. f, PL spectrum at $4 \mathrm{~K}$ for the monolayer TMDs on $\mathrm{SiO}_{2}$, top, versus encapsulated by $h \mathrm{BN}$, bottom ${ }^{51}$. Adapted from ref. ${ }^{4}$, APS (a); ref. $^{49}$, APS (c); ref. $^{50}$, APS (d); ref. ${ }^{51}$, APS (f). 
and a crossover to a power-law temperature dependence is observed. This model agrees well with experimental values for $\mathrm{SiO}_{2}-\mathrm{HfO}_{2}$ encapsulated $\mathrm{MoS}_{2}$ (Fig. 3a, open squares), with $N_{i}=6 \times$ $10^{12} \mathrm{~cm}^{-2}$

Figure $3 b$ shows the measured temperature-dependent mobility of monolayer $\mathrm{MoS}_{2}$ in three different environments: on $\mathrm{SiO}_{2}$ without ${ }^{46}$ (red) and with ${ }^{47}$ (blue) $\mathrm{HfO}_{2}$ encapsulation, and encapsulated with $h \mathrm{BN}^{48}$ (black). On $\mathrm{SiO}_{2}$, the room-T mobility of $\mathrm{MoS}_{2} \sim 10 \mathrm{~cm}^{2} \mathrm{~V}^{-1} \mathrm{~s}^{-1}$, well below the predicted phonon-limited value shown above. Such devices also show a large hysteresis with gate voltage, indicating the presence of mobile charges at the surface ${ }^{54}$. $\mathrm{HfO}_{2}$ encapulation reduces this hysteresis - consistent with passivation of mobile charges - and increases mobility ${ }^{53}$, as does encapsulation with $h \mathrm{BN}^{48}$. In both $\mathrm{HfO}_{2}$ - and $h \mathrm{BN}$-encapsulated devices, a power-law temperature dependence is observed upon cooling, consistent with theory (Fig. 3a), indicating that the mobility is in the intrinsic phonon-limited regime. We note that $h \mathrm{BN}$-encapsulation ( $v s$. $\mathrm{HfO}_{2}$-encapsulation) provides somewhat larger room-T mobility due to its higher optical phonon energy. 4

At low-T, where phonons are inactive, mobility in $\mathrm{SiO}_{2}$-supported devices is limited to $\sim 170$ $\mathrm{cm}^{2} \mathrm{~V}^{-1} \mathrm{~s}^{-1}$. Moreover, at low carrier density such devices show a crossover to a low-T insulating behavior, indicative of disorder-induced localization ${ }^{47} \cdot \mathrm{HfO}_{2}$ encapsulation eliminates the low-T insulating behavior seen at low density, but does not improve the low-T mobility at high carrier density. In contrast, encapsulation within $h \mathrm{BN}$ improves the low-T mobility by roughly one order of magnitude in monolayer $\mathrm{MoS}_{2}$ (see Fig. 3b). Following the theory of Fig. 3a, this increased mobility corresponds to a reduced impurity density of $N_{i} \sim 1 \times 10^{12} \mathrm{~cm}^{-2}$. We note, however, that 
the reduction in impurity density with $h \mathrm{BN}$ encapsulation is still far less than what has been seen for graphene, indicating that either: (1) interfaces in $h \mathrm{BN}-\mathrm{TMD}$ heterostructures are not as clean as for graphene; or (2) TMD crystals possess substantial intrinsic disorder that dominates low-T mobility when extrinsic disorder is reduced. TEM imaging does not find interfacial impurities in $\mathrm{MoS}_{2}-h \mathrm{BN}$ interfaces ${ }^{48}$, arguing against the first scenario. This observation motivates careful examination of intrinsic material quality as discussed below.

The improved performance of $h \mathrm{BN}$-encapsulated $\mathrm{MoS}_{2}$ enables quantum transport measurements in high magnetic fields. In these measurements, the onset of $\mathrm{SdH}$ resistance oscillations decreases as the inverse carrier mobility (quantum scattering time). The first such studies (with $\mu \sim 1,000 \mathrm{~cm}^{2} \mathrm{~V}^{-1} \mathrm{~s}^{-1}$ ) showed the onset of quantum oscillations near $10 \mathrm{~T}^{48}$. More recent data with graphite gate is shown in Figure $3 \mathrm{c}^{49}$. The onset of $\mathrm{SdH}$ oscillations at $\sim 2 \mathrm{~T}$ suggests mobility above 4,000 $\mathrm{cm}^{2} \mathrm{~V}^{-1} \mathrm{~s}^{-1}$ at $\sim 100 \mathrm{mK}$. These oscillations show even-odd behavior, clear evidence of valley Zeeman splitting. Likewise, recent measurements ${ }^{50}$ of hole-doped $\mathrm{WSe}_{2}$ with $\mu \sim 2,000 \mathrm{~cm}^{2} \mathrm{~V}^{-1} \mathrm{~s}^{-1}$ show strong $\mathrm{SdH}$ oscillations with an onset above $5 \mathrm{~T}$, as shown in Fig. 3d. However, fully formed QH states - with zero longitudinal resistance and clear plateaus in Hall resistance - have yet to be observed in monolayer TMDs, highlighting the need for a further reduction in disorder.

Oxidation in air is another source of extrinsic disorder that strongly affects electronic transport in $2 \mathrm{D}$ materials. Encapsulating $2 \mathrm{D}$ monolayers with $h \mathrm{BN}$ in an inert atmosphere provides excellent protection from degradation, allowing air-sensitive samples to be handled easily. This has been widely used to study air-sensitive TMD superconductors such as $\mathrm{NbSe}_{2}$. As shown in 
Fig. 3e, few-layered $\mathrm{NbSe}_{2}$ directly exfoliated on $\mathrm{SiO}_{2}$ does not show superconductivity, and bilayer/trilayer samples show insulating behavior at low-T due to localization from strong disorder ${ }^{55}$. In contrast, $h \mathrm{BN}$-encapsulated $\mathrm{NbSe}_{2}$ is superconducting even in the monolayer limit ${ }^{56}$. This method of using $h \mathrm{BN}$ as a means of isolating samples from oxidation has been expanded to many other layered materials for exploring ferromagnetism $\left(\mathrm{CrI}_{3}\right)^{57}$, or QHE $(\mathrm{BP})^{58}$.

As direct-bandgap semiconductors, monolayer TMDs show efficient photoluminescence (PL), with spectra dominated by strong excitonic resonances. As shown for monolayer TMDs in Fig. 3f, when extrinsic disorder is reduced in monolayer TMDs through $h \mathrm{BN}$ encapsulation and sufficient screening of charge disorder in the the underlying $\mathrm{SiO}_{2}$ substrate, a substantial narrowing of the linewidth is observed ${ }^{51}$. The low-T ( $\left.\sim 4 \mathrm{~K}\right)$ linewidth of $h \mathrm{BN}$ encapsulated samples is less than 2 meV, significantly reduced from the linewidth of $\mathrm{MoSe}_{2}$ on bare $\mathrm{SiO}_{2}{ }^{51,59}$. This reduced linewidth allows for the observation of additional excitonic states at energies close to the highest intensity peaks. In the case of $\mathrm{WSe}_{2}$ this has allowed for the observation and control, via in-plane magnetic fields, of dark excitonic states ${ }^{60}$. These linewidths are even sufficiently narrow enough to allow for the observation of optical transitions between Landau levels with an energy spacing of $\sim 2$ $\mathrm{meV}$ in electrostatically gated monolayer $\mathrm{WSe}_{2}{ }^{61}$. Reduced extrinsic disorder has also facilitated the observation of strong interaction-driven effects such as cyclotron mass renormalization due to electronic-induced doping ${ }^{62}$, biexcitons ${ }^{63}$, and additional many-body states.

\section{Intrinsic disorder in TMDs}

As discussed above, reduction in extrinsic disorder through $h \mathrm{BN}$ encapsulation improves the properties of TMDs, but not to the same degree as observed for graphene, indicating that TMD 
a

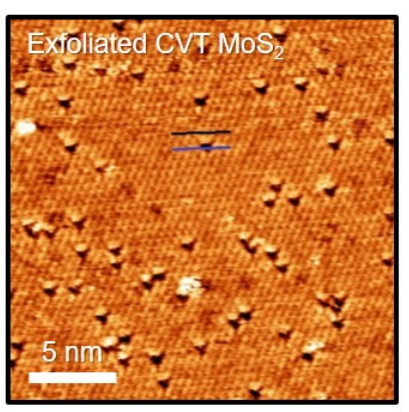

d

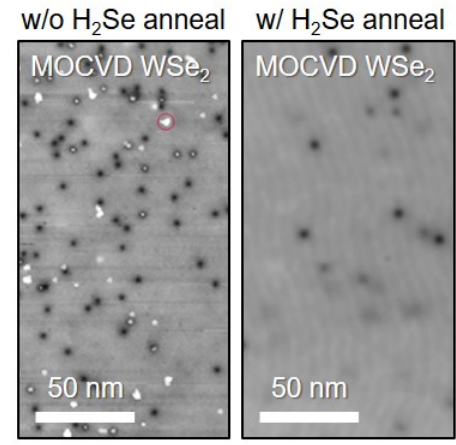

b
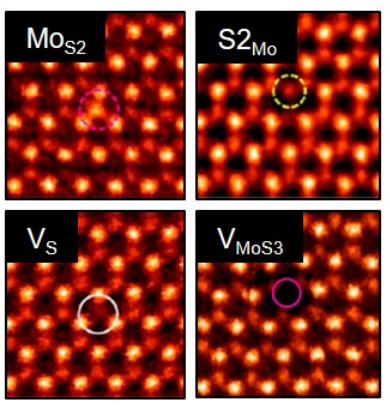

e

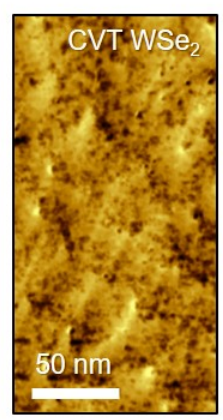

C

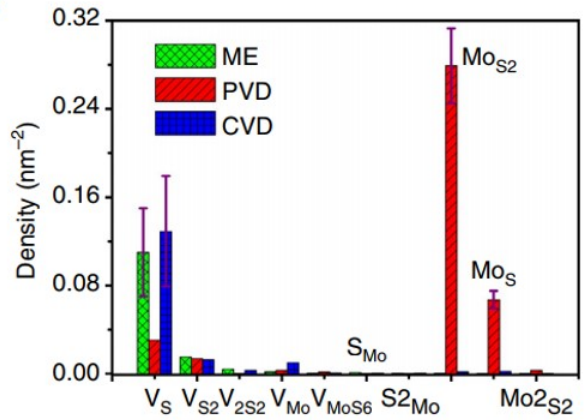

f

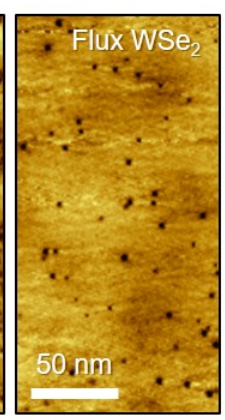

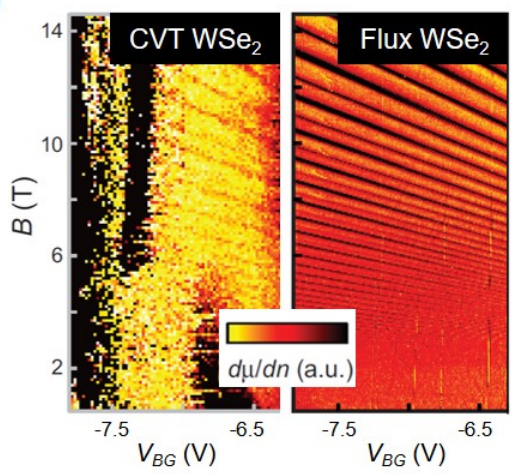

Figure 4 | Intrinsic disorder in TMDs and defect control through growth. a, Topographic STM image of mechanically exfoliated $\mathrm{MoS}_{2}{ }^{64}$. b, ADF-STEM images highlighting each major defect in $\mathrm{MoS}_{2}{ }^{65}$. c, Defect counts for common point defects found in TMDs and for various methods of producing monolayers (mechanical exfoliation, PVD, and CVD $)^{66}$. d, STM image of MOCVD monoalyer $\mathrm{WSe}_{2}$ grown on epitaxial graphene without (left) and with (right) an $\mathrm{H}_{2}$ Se post-growth anneal ${ }^{67}$. e, Topographic STM image of single crystal $\mathrm{WSe}_{2}$ comparing defects for CVT (left) and flux (right) growths. f, Inverse compressibility $\partial \mu / \partial n$ versus back-gate bias and magnetic field $\mathrm{B}$, for CVT (left) and flux (right) grown $\mathrm{WSe}_{2}$, showing an obscured Landau Fan diagram for CVT as compared to flux ${ }^{68}$. Adapted from ref. ${ }^{64}$, Nature Publishing Group (a); ref. ${ }^{65}$, ACS Publications (b); ref. ${ }^{66}$, Nature Publishing Group (c); ref. ${ }^{67}$, ACS Publications (d); ref. ${ }^{68}$, Nature Publishing Group (f). 
crystals do not share the near-perfection of graphene. This is not surprising given that the formation energy for defects in TMDs is much lower than for similar defects in graphene (e.g. $7-8 \mathrm{eV}$ for a graphene vacancy vs. $2.1 \mathrm{eV}$ for a sulfur vacancy in $\left.\mathrm{MoS}_{2}\right)^{65,66}$.

Direct inspection of TMDs by $\operatorname{STM}^{64}$ (Fig. 4a) or ADF-STEM ${ }^{65}$ (Fig. 4b) finds a variety of defect types, including chalcogen vacancies, transition metal vacancies, and antisite defects, in which transition metals occupy chalcogen sites. As one example, Fig. 4c shows the measured density of the major defect types ${ }^{64,66}$ in $\mathrm{MoS}_{2}$ single crystals and films grown by the chemical vapor deposition (CVD) technique. In all of these materials, the defect density (ignoring the presence of impurities) is very high, ranging from mid $10^{12}$ to above $10^{13} \mathrm{~cm}^{-2}$. Moreover, the dominant type of point defect varies depending on the material source: in mined "natural" $\mathrm{MoS}_{2}$ and CVD-grown films, sulfur vacancies can dominate ${ }^{64}$, whereas in synthetic crystals grown by chemical vapor transport (CVT), metal vacancies and antisites dominate ${ }^{69}$. The relative densities of different types of defects determines the crystal doping: for instance, when metal vacancies are dominant, hole (p-type) doping is observed ${ }^{70}$.

Elemental impurities are a second defect class that has to be considered for TMDs. In fact, mined $\mathrm{MoS}_{2}$ can have extremely large impurity concentration of up to $10^{14} \mathrm{~cm}^{-271}$. Artificially grown TMDs from high-purity starting powders show much lower impurity density of $\sim 8 \times 10^{10}$ $\mathrm{cm}^{-2}$, in agreement with the purity of the starting materials ${ }^{72}$. Thus in synthetic TMDs with vacancy density of $\sim 10^{13} \mathrm{~cm}^{-2}$, elemental impurities can be neglected as a source of disorder. However, in materials with substantially lower vacancy density (see below) it will be important to consider both classes of defect. 
Figures $4 \mathrm{~d}$ and $4 \mathrm{e}$ show recent progress in reducing point defect density in TMDs. Figure 4d shows STM images of $\mathrm{WSe}_{2}$ epitaxially grown on graphene by metal-organic chemical vapor deposition (MOCVD) ${ }^{67}$, where the dominant defect types are Se vacancies (black circles) and $\mathrm{W}$ vacancies substituted by Se (grey circles). Annealing in $\mathrm{H}_{2} \mathrm{Se}$ at $800{ }^{\circ} \mathrm{C}$ reduces the density of both defect types by roughly an order of magnitude (to below $10^{12} \mathrm{~cm}^{-2}$ ), and largely eliminates nanoparticles on the surface (white dots). Figure 4e shows STM images of the surface of cleaved $\mathrm{WSe}_{2}$ crystals grown by chemical vapor transport $(\mathrm{CVT})$ - the most commonly utilized technique for TMD crystal growth ${ }^{73}$ - and grown within a liquid chalcogen flux. The flux technique achieves a large reduction in defect density to below $10^{11} \mathrm{~cm}^{-2}$. Initial studies using flux-derived monolayers show dramatic improvements in performance. For instance, the low-T PL intensity in $\mathrm{MoSe}_{2}$ increases by 2 orders of magnitude ${ }^{69}$; and capacitance measurements of the Landau level spectrum of $\mathrm{WSe}_{2}$ show much more detail when flux-grown crystals are used ${ }^{68}$ (Fig. 4f). Finally, we note that an alternative approach to reducing defect density is 'repair' of chalcogen vacancies using thiol chemistry ${ }^{74}$. This approach has been used to achieve large increases in photoluminescence intensity ${ }^{75}$, but does not achieve high low-T mobility consistent with low disorder.

\section{Applications of defects and disorder}

In the sections above, we have focused on the challenge of minimizing disorder in 2D materials. However, disorder in particular crystal defects can also be used to achieve control over electrical, optical, vibrational, magnetic, and chemical properties toward new functionality. Here we briefly discuss some of the most prominent uses of defects in 2D materials. First, substitutional doping can be used for work function control and interface engineering. Layered assembly 

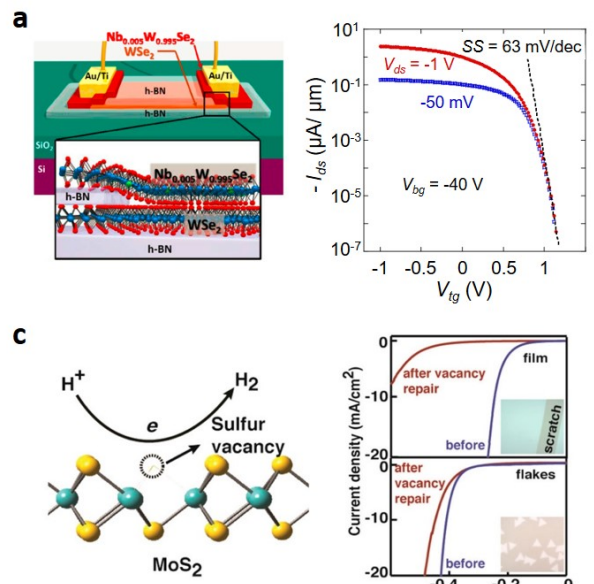

b

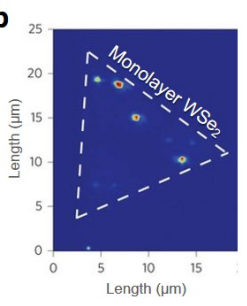

d

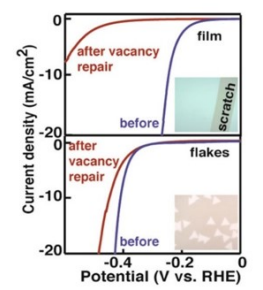

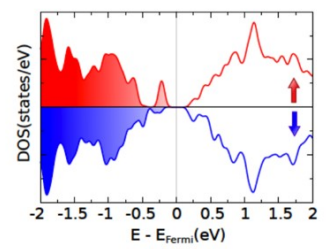
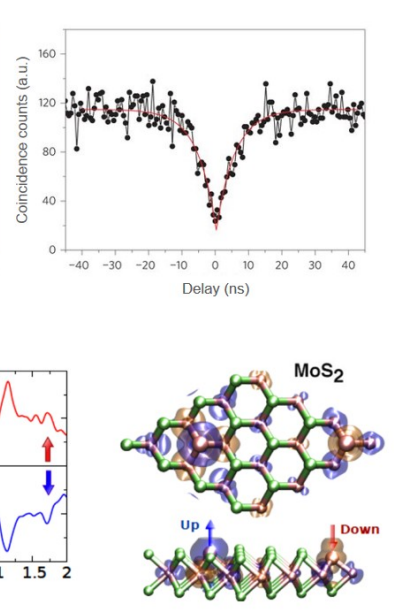

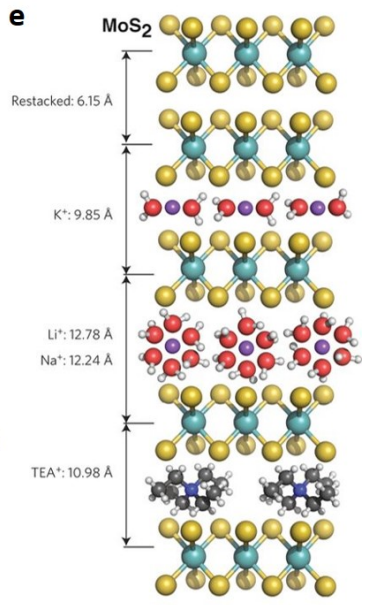

Figure 5 | Applications of defects and disorder in 2D materials. a, Low-resistance ohmic contacts using $\mathrm{Nb}$-doped $\mathrm{WSe}_{2}$ as drain/source contacts ${ }^{76}$. b, PL intensity map and second-order correlation measurement of the PL from single quantum emitters in $\mathrm{WSe}_{2}$ monolayers ${ }^{77}$. The dashed triangle indicates the position of the monolayer $\mathrm{WSe}_{2}$. c, Hydrogen evolution reaction (HER) at the defect sites (sulfur vacancy) of $\mathrm{MoS}_{2}{ }^{78}$. d, Diamagnetism and ferromagnetism have been observed in of $\mathrm{MoS}_{2}{ }^{79}$. e, Schematics of restacked nonintercalated and intercalated $\mathrm{MoS}_{2}$ by alkali ions $\left(\mathrm{Li}^{+}, \mathrm{Na}^{+}, \mathrm{K}^{+}\right)$and TEA ${ }^{+80}$. Adapted from ref. ${ }^{76}$, ACS Publications (a); ref. ${ }^{77}$, Nature Publishing Group (b); ref. ${ }^{78}$, ACS Publications (c); ref. $^{79}$ (d); ref. ${ }^{80}$ Nature Publishing Group (e). 
of doped 2D semiconductors circumvents problems such as work function mismatch and Fermi level pinning that create large Schottky barriers in metal-TMD contacts ${ }^{81}$. Figure 5a shows that $\mathrm{Nb}$-doped $\mathrm{WSe}_{2}$ functions as a near-ideal $p$-type contact to intrinsic $\mathrm{WSe}_{2}{ }^{76}$, achieving contact resistance of $\sim 0.3 \mathrm{k} \Omega \mu \mathrm{m}$ and steep subthreshold slope. Doping of $2 \mathrm{D}$ materials has been extensively reviewed ${ }^{82}$.

Individual defects in $2 \mathrm{D}$ materials can also act as optically active sites. Figure $5 \mathrm{~b}$ shows the single-photon emission from localized defects in $\mathrm{WSe}_{2}$ monolayers ${ }^{77}$. The measured autocorrelation function $\mathrm{g}^{2}(\tau)$ of the emission shows a dip below 0.5 at zero delay time $(\tau=0)$, indicating that each defect acts as a single-photon emitter. Tunable single photon emission from semiconducting TMDs has been reported ${ }^{83}$, and $h \mathrm{BN}$-based quantum emitters are optically active at room temperature, an important attribute for applications in quantum information ${ }^{84}$. Although the exact mechanism is still uncertain, this emission is likely due to defect sites that are activated in the presence of strain. Greater control over optically active defects, coupled with precise charge injection in van der Waals heterostructures, will allow TMDs to be used for electrically controlled quantum emitters for quantum information processing.

Defects in TMDs also hold promise as catalytic sites that can replace expensive noble metal catalysts in the hydrogen evolution reaction (HER) and other processes. $\mathrm{MoS}_{2}$ has been demonstrated as an effective catalyst for HER at a moderate overpotential of $0.1-0.2 \mathrm{~V}^{85}$. Catalysis occurs at defects including edges, sulfur vacancies, and grain boundaries ${ }^{78}$ (Fig. 5c). Changing the sulfur vacancy density simultaneously tunes the density of catalytic sites and doping in the sheet, allowing for optimization of both catalytic activity and charge injection to the TMD layer ${ }^{78}$. 
Defects in TMDs can give rise to diamagnetism, ferromagnetism, and spin-valley coupling applications. Recent experimental work has shown that point defects in Mo-based TMDs can be magnetic, as confirmed by calculation of the spin-polarized density of states for an antisite defect (Mo at the chalcogen site), shown in Fig. $5 \mathrm{~d}$ for $\mathrm{MoTe}_{2}$. These spins couple antiferromagnetically to nearby Mo atoms and can show long-range ordering ${ }^{79}$. Spin polarized first-principles calculations suggest that one-dimensional edges of two-dimensional $\mathrm{MoS}_{2}$ or $\mathrm{WS}_{2}$ carry non-zero net magnetic moments associated with unpaired electrons ${ }^{86}$. Atomic defects also give unconventional selection rules for intervalley quasiparticle scattering in 2D TMDs valleytronics ${ }^{87}$.

Interface chemistry approaches in $2 \mathrm{D}$ materials seek to manipulate the electrochemical behaviour of layered systems, and open new pathways to control the charge density in electronic and optoelectronic devices. For instance, it has been shown that intercalation by organolithium chemistry in $2 \mathrm{H}$-TMDs stabilizes a metallic $1 \mathrm{~T}$ phase via strong electron doping (see schematic image, Fig. $5 \mathrm{e}^{80}$ ). This method can be further applied to phase-engineer contacts, reducing contact resistance to $200-300 \Omega \mu \mathrm{m}$ in few-layered $2 \mathrm{H}-\mathrm{MoS}_{2}$, comparable to the best contacts produced through more intricate fabrication methods ${ }^{88}$.

\section{Outlook}

Reducing disorder in 2D materials has enabled stunning scientific advances and progress toward technological applications, providing strong motivation to continue these efforts. Toward this end, each materials system faces distinct challenges. In graphene devices, the remaining sources of extrinsic disorder must be identified and further reduced. One possible source is environmental contaminants such as water vapour and hydrocarbons which are not completely eliminated from 
interfaces during heterostructure fabrication. A second possible source is defects in $h \mathrm{BN}$. There has been little work beyond $h \mathrm{BN}$ synthesis ${ }^{23}$ in reducing defects and increasing grain size in $h \mathrm{BN}$ single crystals. More work is needed in this area, as well as in development of methods to quickly assess defect density in $h \mathrm{BN}$. In $h \mathrm{BN}$-encapsulated 2D semiconductors, on the other hand, intrinsic defects of 2D semiconductors are the dominant source of disorder. The need for careful characterization of materials quality - which can vary widely with with synthesis technique and from batch to batch - should be emphasized in ongoing studies of novel phenomena in TMDs. Further progress in the field will require reduction in defect density in both single crystals and large-area films of every material under study. In order to achieve rapid progress toward this goal, it will be important to develop facile techniques for in situ characterization of materials quality. However, this effort is still impeded by a lack of basic understanding of how atomic defects affect easilymeasured bulk properties.

Realizing the potential of 2D materials in applications will require development of materials synthesis and manufacturing techniques that can achieve low disorder on a large scale. A specific challenge is to achieve low extrinsic disorder in large-area films. Toward this end, significant progress has been made in the synthesis of graphene and TMD films at the wafer scale, as well as in the development of clean, wafer-scale transfers of monolayer films ${ }^{89-91}$. Scaling up encapsulation approaches used to reduce disorder in exfoliated flakes will require synthesis of multilayer $h \mathrm{BN}$ films with high uniformity and large grain size, which has proven to be extremely challenging to date $^{92}$. Alternatively, other dielectric materials may provide sufficient performance: for example, many layered metal oxides to (i.e. $\mathrm{MoO}_{3}, \mathrm{~V}_{2} \mathrm{O}_{5}$, etc.) with bandgaps in excess of $3.5 \mathrm{eV}$ have yet to 
be extensively explored as as dielectrics for graphene and 2D semiconductors ${ }^{93}$. The important role of defects and dopants in 2D materials highlights another gap in current capabilities: in conventional semiconductors dopants can be patterned using ion implantation, allowing precise control over dopant density required for high-performance electronic and optoelectronic devices. While techniques such as plasma treatment and wet chemical treatment have demonstrated local doping of 2D semiconductors, no current technique provides precise local control over doping combined with low disorder and good stability. Finally, we note that improving the intrinsic quality of 2D materials will improve fundamental understanding of the behavior of defects by permitting careful study of well-characterized defects embedded in high-quality crystals.

\section{References}

1. Huang, P. Y. et al. Grains and grain boundaries in single-layer graphene atomic patchwork quilts. Nature 469, 389-392 (2011).

2. Shin, B. G. et al. Indirect Bandgap Puddles in Monolayer $\mathrm{MoS}_{2}$ by Substrate-Induced Local Strain. Advanced Materials 28, 9378-9384 (2016).

3. Favron, A. et al. Photooxidation and quantum confinement effects in exfoliated black phosphorus. Nature Materials 14, 826-832 (2015).

4. Ma, N. \& Jena, D. Charge Scattering and Mobility in Atomically Thin Semiconductors. Phys. Rev. X 4, 011043 (2014). 
5. Sachs, B., Wehling, T. O., Katsnelson, M. I. \& Lichtenstein, A. I. Midgap states and band gap modification in defective graphene/h-BN heterostructures. Phys. Rev. B 94, 224105 (2016).

6. Wang, H., Zhang, C. \& Rana, F. Ultrafast Dynamics of Defect-Assisted ElectronHole Recombination in Monolayer $\mathrm{MoS}_{2}$. Nano Lett. 15, 339-345 (2015).

7. Tsen, A. W. et al. Tailoring Electrical Transport Across Grain Boundaries in Polycrystalline Graphene. Science 336, 1143-1146 (2012).

8. Wang, D. et al. Bandgap broadening at grain boundaries in single-layer $\mathrm{MoS}_{2}$. Nano Res. 11, 6102-6109 (2018).

9. Hus, S. M. \& Li, A.-P. Spatially-resolved studies on the role of defects and boundaries in electronic behavior of 2d materials. Progress in Surface Science 92, 176-201 (2017).

10. Novoselov, K. S. et al. Two-dimensional gas of massless Dirac fermions in graphene. Nature 438, 197-200 (2005).

11. Martin, J. et al. Observation of electronhole puddles in graphene using a scanning singleelectron transistor. Nature Physics 4, 144-148 (2008).

12. Xue, J. et al. Scanning tunnelling microscopy and spectroscopy of ultra-flat graphene on hexagonal boron nitride. Nature Materials 10, 282-285 (2011).

13. Decker, R. et al. Local Electronic Properties of Graphene on a BN Substrate via Scanning Tunneling Microscopy. Nano Lett. 11, 2291-2295 (2011). 
14. Zhang, Y., Brar, V. W., Girit, C., Zettl, A. \& Crommie, M. F. Origin of spatial charge inhomogeneity in graphene. Nature Physics 5, 722-726 (2009).

15. Chen, J.-H. et al. Charged-impurity scattering in graphene. Nature Physics 4, 377-381 (2008).

16. Adam, S., Hwang, E. H., Galitski, V. M. \& Sarma, S. D. A self-consistent theory for graphene transport. PNAS 104, 18392-18397 (2007).

17. Geim, A. K. \& Grigorieva, I. V. Van der Waals heterostructures. Nature 499, 419-425 (2013).

18. Du, X., Skachko, I., Barker, A. \& Andrei, E. Y. Approaching ballistic transport in suspended graphene. Nature Nanotechnology 3, 491-495 (2008).

19. Bolotin, K. I., Ghahari, F., Shulman, M. D., Stormer, H. L. \& Kim, P. Observation of the fractional quantum Hall effect in graphene. Nature 462, 196-199 (2009).

20. Feldman, B. E., Krauss, B., Smet, J. H. \& Yacoby, A. Unconventional Sequence of Fractional Quantum Hall States in Suspended Graphene. Science 337, 1196-1199 (2012).

21. Jr, J. V. et al. Transport spectroscopy of symmetry-broken insulating states in bilayer graphene. Nature Nanotechnology 7, 156-160 (2012).

22. Nam, Y., Ki, D.-K., Soler-Delgado, D. \& Morpurgo, A. F. Electronhole collision limited transport in charge-neutral bilayer graphene. Nature Physics 13, 1207-1214 (2017).

23. Taniguchi, T. \& Watanabe, K. Synthesis of high-purity boron nitride single crystals under high pressure by using BaBN solvent. Journal of Crystal Growth 303, 525-529 (2007). 
24. Dean, C. R. et al. Boron nitride substrates for high-quality graphene electronics. Nature Nanotechnology 5, 722-726 (2010).

25. Garcia, A. G. F. et al. Effective Cleaning of Hexagonal Boron Nitride for Graphene Devices. Nano Lett. 12, 4449-4454 (2012).

26. Ponomarenko, L. A. et al. Tunable metalinsulator transition in double-layer graphene heterostructures. Nature Physics 7, 958-961 (2011).

27. Wang, L. et al. One-Dimensional Electrical Contact to a Two-Dimensional Material. Science 342, 614-617 (2013).

28. Osvald, J. On barrier height inhomogeneities at polycrystalline metal-semiconductor contacts. Solid-State Electronics 35, 1629-1632 (1992).

29. Zibrov, A. A. et al. Tunable interacting composite fermion phases in a half-filled bilayergraphene Landau level. Nature 549, 360-364 (2017).

30. Adam, S. \& Das Sarma, S. Transport in suspended graphene. Solid State Communications 146, 356-360 (2008).

31. Du, X., Skachko, I., Duerr, F., Luican, A. \& Andrei, E. Y. Fractional quantum Hall effect and insulating phase of Dirac electrons in graphene. Nature 462, 192-195 (2009).

32. Banszerus, L. et al. Ballistic Transport Exceeding $28 \mu \mathrm{m}$ in CVD Grown Graphene. Nano Lett. 16, 1387-1391 (2016). 
33. Wang, L. et al. Evidence for a fractional fractal quantum Hall effect in graphene superlattices. Science 350, 1231-1234 (2015).

34. Chen, S. et al. Competing Fractional Quantum Hall and Electron Solid Phases in Graphene. Phys. Rev. Lett. 122, 026802 (2019).

35. Zibrov, A. A. et al. Even-denominator fractional quantum hall states at an isospin transition in monolayer graphene. Nature Physics 14, 930-935 (2018).

36. Zeng, Y. et al. Ultra-high quality magnetotransport in graphene using the edge-free Corbino geometry. arXiv:1805.04904 [cond-mat] (2018).

37. Schreiber, K. A. et al. Onset of quantum criticality in the topological-to-nematic transition in a two-dimensional electron gas at filling factor $\nu=5 / 2$. Phys. Rev. B 96, 041107 (2017).

38. Shi, Q. et al. Microwave photoresistance in an ultra-high-quality GaAs quantum well. Phys. Rev. B 93, 121305 (2016).

39. Pan, W., Baldwin, K. W., West, K. W., Pfeiffer, L. N. \& Tsui, D. C. Fractional quantum Hall effect at Landau level filling $\nu=4 / 11$. Phys. Rev. B 91, 041301 (2015).

40. Koulakov, A. A., Fogler, M. M. \& Shklovskii, B. I. Charge Density Wave in Two-Dimensional Electron Liquid in Weak Magnetic Field. Phys. Rev. Lett. 76, 499-502 (1996).

41. Forsythe, C. et al. Band structure engineering of $2 \mathrm{~d}$ materials using patterned dielectric superlattices. Nature Nanotechnology 13, 566-571 (2018). 
42. Li, J. et al. Negative Coulomb Drag in Double Bilayer Graphene. Phys. Rev. Lett. 117, 046802 (2016).

43. Dean, C. R. et al. Hofstadters butterfly and the fractal quantum Hall effect in moir superlattices. Nature 497, 598-602 (2013).

44. Yu, G. L. et al. Hierarchy of Hofstadter states and replica quantum Hall ferromagnetism in graphene superlattices. Nature Physics 10, 525-529 (2014).

45. Cao, Y. et al. Unconventional superconductivity in magic-angle graphene superlattices. Nature 556, 43-50 (2018).

46. Baugher, B. W. H., Churchill, H. O. H., Yang, Y. \& Jarillo-Herrero, P. Intrinsic Electronic Transport Properties of High-Quality Monolayer and Bilayer $\mathrm{MoS}_{2}$. Nano Lett. 13, 42124216 (2013).

47. Radisavljevic, B. \& Kis, A. Mobility engineering and a metalinsulator transition in monolayer $\mathrm{MoS}_{2}$. Nature Materials 12, 815-820 (2013).

48. Cui, X. et al. Multi-terminal transport measurements of $\mathrm{MoS}_{2}$ using a van der Waals heterostructure device platform. Nature Nanotechnology 10, 534-540 (2015).

49. Pisoni, R. et al. Interactions and magnetotransport through spin-valley coupled Landau levels in monolayer $\mathrm{MoS}_{2}$. Phys. Rev. Lett. 121, 247701 (2018). 
50. Fallahazad, B. et al. Shubnikov-de Haas Oscillations of High-Mobility Holes in Monolayer and Bilayer $\mathrm{WSe}_{2}$ : Landau Level Degeneracy, Effective Mass, and Negative Compressibility. Phys. Rev. Lett. 116, 086601 (2016).

51. Cadiz, F. et al. Excitonic Linewidth Approaching the Homogeneous Limit in $\mathrm{MoS}_{2}$-Based van der Waals Heterostructures. Phys. Rev. X 7, 021026 (2017).

52. Wang, Q. H., Kalantar-Zadeh, K., Kis, A., Coleman, J. N. \& Strano, M. S. Electronics and optoelectronics of two-dimensional transition metal dichalcogenides. Nature Nanotechnology 7, 699-712 (2012).

53. Yu, Z. et al. Realization of Room-Temperature Phonon-Limited Carrier Transport in Monolayer $\mathrm{MoS}_{2}$ by Dielectric and Carrier Screening. Advanced Materials 28, 547-552 (2016).

54. Late, D. J., Liu, B., Matte, H. S. S. R., Dravid, V. P. \& Rao, C. N. R. Hysteresis in Single-Layer $\mathrm{MoS}_{2}$ Field Effect Transistors. ACS Nano 6, 5635-5641 (2012).

55. Staley, N. E. et al. Electric field effect on superconductivity in atomically thin flakes of $\mathrm{NbSe}_{2}$. Phys. Rev. B 80, 184505 (2009).

56. Xi, X. et al. Ising pairing in superconducting $\mathrm{NbSe}_{2}$ atomic layers. Nature Physics 12, 139143 (2016).

57. Huang, B. et al. Layer-dependent ferromagnetism in a van der Waals crystal down to the monolayer limit. Nature 546, 270-273 (2017). 
58. Li, L. et al. Quantum Hall effect in black phosphorus two-dimensional electron system. Nature Nanotechnology 11, 593-597 (2016).

59. Ajayi, O. A. et al. Approaching the intrinsic photoluminescence linewidth in transition metal dichalcogenide monolayers. 2D Mater. 4, 031011 (2017).

60. Zhang, X.-X. et al. Magnetic brightening and control of dark excitons in monolayer $\mathrm{WSe}_{2}$. Nature Nanotechnology 12, 883-888 (2017).

61. Movva, H. C. et al. Density-Dependent Quantum Hall States and Zeeman Splitting in Monolayer and Bilayer WSe ${ }_{2}$. Phys. Rev. Lett. 118, 247701 (2017).

62. Wang, Z., Shan, J. \& Mak, K. F. Valley- and spin-polarized Landau levels in monolayer WSe ${ }_{2}$. Nature Nanotechnology 12, 144-149 (2017).

63. You, Y. et al. Observation of biexcitons in monolayer $\mathrm{WSe}_{2}$. Nature Physics 11, 477-481 (2015).

64. Vancs, P. et al. The intrinsic defect structure of exfoliated $\mathrm{MoS}_{2}$ single layers revealed by Scanning Tunneling Microscopy. Scientific Reports 6, 29726 (2016).

65. Zhou, W. et al. Intrinsic Structural Defects in Monolayer Molybdenum Disulfide. Nano Lett. 13, 2615-2622 (2013).

66. Hong, J. et al. Exploring atomic defects in molybdenum disulphide monolayers. Nature Communications 6, 6293 (2015). 
67. Lin, Y.-C. et al. Realizing Large-Scale, Electronic-Grade Two-Dimensional Semiconductors. ACS Nano 12, 965-975 (2018).

68. Gustafsson, M. V. et al. Ambipolar Landau levels and strong band-selective carrier interactions in monolayer $\mathrm{WSe}_{2}$. Nature Materials 17, 411-415 (2018).

69. Edelberg, D. et al. Hundredfold Enhancement of Light Emission via Defect Control in Monolayer Transition-Metal Dichalcogenides. arXiv:1805.00127 [cond-mat] (2018).

70. Zhang, S. et al. Defect Structure of Localized Excitons in a WSe $\mathrm{W}_{2}$ Monolayer. Phys. Rev. Lett. 119, $046101(2017)$.

71. Addou, R. et al. Impurities and Electronic Property Variations of Natural $\mathrm{MoS}_{2}$ Crystal Surfaces. ACS Nano 9, 9124-9133 (2015).

72. Addou, R. \& Wallace, R. M. Surface Analysis of WSe ${ }_{2}$ Crystals: Spatial and Electronic Variability. ACS Appl. Mater. Interfaces 8, 26400-26406 (2016).

73. Qiu, H. et al. Hopping transport through defect-induced localized states in molybdenum disulphide. Nature Communications 4, 2642 (2013).

74. Yu, Z. et al. Towards intrinsic charge transport in monolayer molybdenum disulfide by defect and interface engineering. Nature Communications 5, 5290 (2014).

75. Amani, M. et al. Near-unity photoluminescence quantum yield in $\mathrm{MoS}_{2}$. Science 350, 10651068 (2015). 
76. Chuang, H.-J. et al. Low-Resistance 2d/2d Ohmic Contacts: A Universal Approach to HighPerformance $\mathrm{WSe}_{2}, \mathrm{MoS}_{2}$, and $\mathrm{MoSe}_{2}$ Transistors. Nano Lett. 16, 1896-1902 (2016).

77. He, Y.-M. et al. Single quantum emitters in monolayer semiconductors. Nature Nanotechnology 10, 497-502 (2015).

78. Li, G. et al. All The Catalytic Active Sites of $\mathrm{MoS}_{2}$ for Hydrogen Evolution. J. Am. Chem. Soc. 138, 16632-16638 (2016).

79. Guguchia, Z. et al. Magnetism in semiconducting molybdenum dichalcogenides. Science Advances 4, 3672 (2018).

80. Acerce, M., Voiry, D. \& Chhowalla, M. Metallic $1 \mathrm{~T}$ phase $\mathrm{MoS}_{2}$ nanosheets as supercapacitor electrode materials. Nature Nanotechnology 10, 313-318 (2015).

81. Das, S., Chen, H.-Y., Penumatcha, A. V. \& Appenzeller, J. High Performance Multilayer $\mathrm{MoS}_{2}$ Transistors with Scandium Contacts. Nano Lett. 13, 100-105 (2013).

82. Zhao, Y. et al. Doping, Contact and Interface Engineering of Two-Dimensional Layered Transition Metal Dichalcogenides Transistors. Advanced Functional Materials 27, 1603484 (2017).

83. Chakraborty, C., Kinnischtzke, L., Goodfellow, K. M., Beams, R. \& Vamivakas, A. N. Voltage-controlled quantum light from an atomically thin semiconductor. Nature Nanotechnology 10, 507-511 (2015). 
84. Tran, T. T., Bray, K., Ford, M. J., Toth, M. \& Aharonovich, I. Quantum emission from hexagonal boron nitride monolayers. Nature Nanotechnology 11, 37-41 (2016).

85. Hinnemann, B. et al. Biomimetic Hydrogen Evolution: $\mathrm{MoS}_{2}$ Nanoparticles as Catalyst for Hydrogen Evolution. J. Am. Chem. Soc. 127, 5308-5309 (2005).

86. Zhang, J. et al. Magnetic Molybdenum Disulfide Nanosheet Films. Nano Lett. 7, 2370-2376 (2007).

87. Kaasbjerg, K., Martiny, J. H. J., Low, T. \& Jauho, A.-P. Symmetry-forbidden intervalley scattering by atomic defects in monolayer transition-metal dichalcogenides. Phys. Rev. B 96, 241411 (2017).

88. Kappera, R. et al. Phase-engineered low-resistance contacts for ultrathin $\mathrm{MoS}_{2}$ transistors. Nature Materials 13, 1128-1134 (2014).

89. Kang, K. et al. High-mobility three-atom-thick semiconducting films with wafer-scale homogeneity. Nature 520, 656-660 (2015).

90. Kang, K. et al. Layer-by-layer assembly of two-dimensional materials into wafer-scale heterostructures. Nature 550, 229-233 (2017).

91. Shim, J. et al. Controlled crack propagation for atomic precision handling of wafer-scale two-dimensional materials. Science 362, 665-670 (2018).

92. Jana, M. \& Singh, R. N. Progress in CVD synthesis of layered hexagonal boron nitride with tunable properties and their applications. International Materials Reviews 63, 162-203 (2018). 
93. Kalantar-zadeh, K. et al. Two dimensional and layered transition metal oxides. Applied Materials Today 5, 73-89 (2016).

Acknowledgements We would like to acknowledge Matthew Yankowitz and J.I.A. Li for many discussions involving graphene and graphene devices. This work was supported the NSF MRSEC program through Columbia in the Center for Precision Assembly of Superstratic and Superatomic Solids (DMR-1420634). S.H.C. was supported by the Postdoctoral Research Program of Sungkyunkwan University (2016).

Competing Interests The authors declare that they have no competing financial interests.

Correspondence Correspondence and requests for materials should be addressed to J.H. (email: jh2228@columbia.edu). 